\title{
Speeding Up Back-Propagation Neural Networks
}

\author{
Mohammed A. Otair \\ Jordan University of Science \\ and Technology, \\ Irbed, Jordan
}

otair@just.edu.jo

\author{
Walid A. Salameh \\ Princess Summaya University \\ for Science and Technology, \\ Amman, Jordan
}

walid@psut.edu.jo

\begin{abstract}
There are many successful applications of Backpropagation (BP) for training multilayer neural networks. However, it has many shortcomings. Learning often takes long time to converge, and it may fall into local minima. One of the possible remedies to escape from local minima is by using a very small learning rate, which slows down the learning process. The proposed algorithm presented in this study used for training depends on a multilayer neural network with a very small learning rate, especially when using a large training set size. It can be applied in a generic manner for any network size that uses a backpropgation algorithm through an optical time (seen time). The paper describes the proposed algorithm, and how it can improve the performance of backpropagation (BP). The feasibility of proposed algorithm is shown through out number of experiments on different network architectures.
\end{abstract}

Keywords: Neural Networks, Backpropagation, Modified backprpoagation, Non-Linear function, Optical Algorithm.

\section{Introduction}

The Backpropagation (BP) algorithm (Rumelhart, Hinton, \& Williams, 1986; Rumelhart, Durbin, Golden, \& Chauvin, 1992) is perhaps the most widely used supervised training algorithm for multi-layered feedforward neural networks. However, in some cases, the standard Backpropagation takes unendurable time to adapt the weights between the units in the network to minimize the mean squared errors between the desired outputs and the actual network outputs (Callan, 1999; Carling, 1992; Freeman, \&, Skapura, 1992; Hakin, 1999; Maureen, 1993).

There has been much research proposed to improve this algorithm; some of this research was based on the adaptive learning parameters, e.g. the Quickprop (Fahlman, 1988), the RPROP (Riedmiller, \& Braun, 1993), delta-bar-delta rule (Jacobs, 1988), and Extended delta-bar-delta rule (Minai, 1990). Combinations of different techniques can often lead to an improvement in global optimization methods (Hagan, 1996; Lee, 1991).

This paper presents an optical backpropagation (OBP) algorithm, with analysis of its benefits. An

Material published as part of these proceedings, either on-line or in print, is copyrighted by Informing Science. Permission to make digital or paper copy of part or all of these works for personal or classroom use is granted without fee provided that the copies are not made or distributed for profit or commercial advantage AND that copies 1) bear this notice in full and 2) give the full citation on the first page. It is permissible to abstract these works so long as credit is given. To copy in all other cases or to republish or to post on a server or to redistribute to lists requires specific permission from the publisher at Publisher@InformingScience.org
OBP algorithm is designed to overcome some of the problems associated with standard BP training using nonlinear function, which applied on the output units. One of the important aspects of the proposed algorithm is its ability to escape from local minima with high speed of convergence during the training period. In order to 
evaluate the performance of the proposed algorithm, simulations are carried out on different network architectures, and the results are compared with results obtained from standard BP.

This paper is divided into four sections. In section 2, we will introduce the standard Backpropagation $(B P)$. In section 3, our extension of the non-linear function of the BP is declared. In section 4, a comparative study will be done over different network architectures. Finally, the conclusions are presented in section 5 .

\section{Standard Backpropagation (BP)}

The Backpropagation $B P$ learns a predefined set of output example pairs by using a two-phase propagate adapts cycle. As seen in Figure 1, after an input pattern has been applied as a stimulus to first layer of network units, it is propagated through each upper layer until an output is gener-

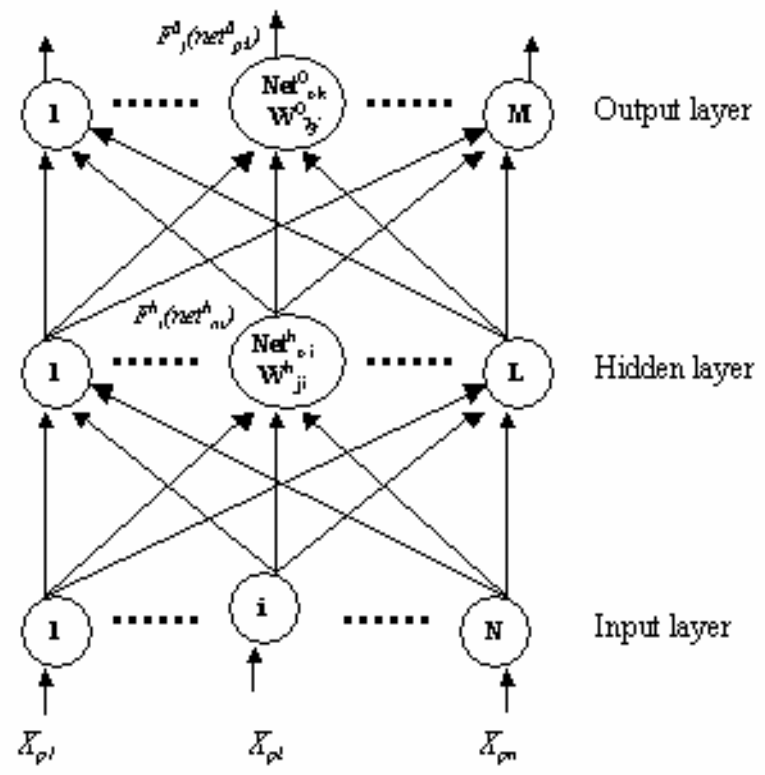

Figure 1 - The Three-layer BP Architecture ated. This output pattern is then compared to the desired output, and an error signal is computed for each output unit.

The signals are then transmitted backward from the output layer to each unit in the intermediate layer that contributes directly to the output. However, each unit in the intermediate layer receives only a portion of the total error signal, based roughly on the relative contribution the unit made to the original output. This process repeats, layer by layer, until each unit in the network has received an error signal that describes its relative contribution to the total error.

We follow Freeman and Skapura's book (1992) to describe the procedure of training feedforward neural networks using the backpropagation algorithm. The detailed formulas are described in the Appendix.

\section{Optical Backpropagation (OBP)}

In this section, the adjustment of the new algorithm OBP (Otair \& Salameh, 2004) will be described at which it would improve the performance of the $B P$ algorithm. The convergence speed of the learning process can be improved significantly by $O B P$ through adjusting the error, which will be transmitted backward from the output layer to each unit in the intermediate layer.

In $B P$, the error at a single output unit is defined as:

$$
\delta^{0}{ }_{\mathrm{pk}}=\left(\mathrm{Y}_{\mathrm{pk}}-\mathrm{O}_{\mathrm{pk}}\right)
$$

Where the subscript " $P$ "refers to the $p_{t h}$ training vector, and " $K$ "refers to the $k_{t h}$ output unit. In this case, $Y_{p k}$ is the desired output value, and $O_{p k}$ is the actual output from $k_{t h}$ unit, then $\delta_{\mathrm{pk}}$ will propagate backward to update the output-layer weights and the hidden-layer weights.

While the error at a single output unit in adjusted $O B P$ will be as: 


$$
\begin{aligned}
& \mathrm{New} \delta \mathrm{o} p \mathrm{pk}=\left(1+\mathrm{e}^{\left(\mathrm{Y}_{\mathrm{pk}}-\mathrm{O}_{\mathrm{pk}}\right)^{2}},\right. \\
&, \text { if }\left(Y_{p k}-O_{p k}\right)>=\text { zero. } \\
& \mathrm{New} \delta^{\mathrm{O}} \mathrm{pk}=-\left(1+\mathrm{e}^{\left(\mathrm{Y}_{\mathrm{pk}}-\mathrm{O}_{\mathrm{pk}}\right)^{2}}\right) \\
&, \text { if }\left(Y_{p k}-O_{p k}\right)<\text { zero. }
\end{aligned}
$$

Where $N e w \delta_{\mathrm{pk}}^{\circ}$ is considered as the new proposed in the OBP algorithm.

An $O B P$ uses two forms of $N e w \delta_{p k}^{\circ}$, because the $\exp$ function always returns zero or positive values (and the adapts operation for many output units need to decrease the actual outputs rather than increasing it). This $N e w \delta_{p k}^{\circ}$ will minimize the errors of each output unit more quickly than the old $\delta_{\mathrm{pk}}^{\circ}$, and the weights on certain units change very large from their starting values.

\section{The steps of an OBP:}

1. Apply the input example to the input units.

2. Calculate the net-input values to the hidden layer units.

3. Calculate the outputs from the hidden layer.

4. Calculate the net-input values to the output layer units.

5. Calculate the outputs from the output units.

6. Calculate the error term for the output units, but replace $N e w \delta^{\circ} p k$ with $\delta^{\circ} p k$ (in all equations in appendix).

7. Calculate the error term for the output units, using $N e w \delta^{\circ} \mathrm{pk}$, also.

8. Update weights on the output layer.

9. Update weights on the hidden layer.

10. Repeat steps from step $\mathbf{1}$ to step $\mathbf{9}$ until the error $\left(\mathrm{Y}_{\mathrm{pk}}-\mathrm{O}_{\mathrm{pk}}\right)$ is acceptably small for each training vector pairs.

\section{Proof:}

The output of $B P$ and $O B P$ for any output unit must be equal, if the $B P$ output units multiply it by Factor $(A)$, where $(A)$ is defined as follows:

$$
\begin{aligned}
& \mathrm{A} \bullet \frac{1}{1+\mathrm{e}^{-\mathrm{X}}}=\left(1+\mathrm{e}^{\left(\mathrm{Y}_{\mathrm{pk}}-\mathrm{O}_{\mathrm{pk}}\right)^{2}}\right) \\
& \mathrm{A}=\left(1+\mathrm{e}^{\left(\mathrm{Y}_{\mathrm{pk}}-\mathrm{O}_{\mathrm{pk}}\right)^{2}}\right) \bullet 1+\mathrm{e}^{-\mathrm{X}}
\end{aligned}
$$

The sigmoid function for each output unit in $B P$ must be equal to the $N e w \delta_{p k}^{\circ}$ in $O B P$ through multiplying it by this Factor.

There is another way to find the factor $(A)$ using the following assumptions: 


\section{Assumption 1:}

$$
\mathrm{A} 1 \bullet \frac{1}{1+\mathrm{e}^{-\mathrm{X}}}=\frac{1}{\left(1+\mathrm{e}^{\mathrm{Y}^{-\mathrm{O}}}{ }_{\mathrm{pk}}\right)}
$$

In other words, $O B P$ uses a sigmoid function on each error of each output unit $\left(\mathrm{Y}_{\mathrm{pk}}-\mathrm{O}_{\mathrm{pk}}\right)$, and it assumes that if the sigmoid function (on the left side in equation 6 ) is multiplied by (A1), it must be equal to those sigmoid function which applied on the error for the output units, then:

$$
\mathrm{A} 1=\frac{1+\mathrm{e}^{-\mathrm{x}}}{\left(1+\mathrm{e}^{\mathrm{Y}_{\mathrm{pk}}-\mathrm{O}_{\mathrm{pk}}}\right)}
$$

\section{* Assumption 2:}

An $O B P$ assumes that if the sigmoid function - which is applied on the error of output units - is multiplied by $(A 2)$, then it must be equal to $N e w \delta_{\text {pk: }}^{\circ}$

$$
\mathrm{A} 2 \cdot \frac{1}{\left(1+\mathrm{e}^{\mathrm{Y} k}-\mathrm{O}_{\mathrm{pk}}\right)}=\left(1+\mathrm{e}^{\left(\mathrm{Y}_{\mathrm{pk}}-\mathrm{O}_{\mathrm{pk}}\right)^{2}}\right) \quad \text { (8) } \mathrm{A} 2=\left(1+\mathrm{e}^{\left(\mathrm{Y}_{\mathrm{pk}}-\mathrm{O}_{\mathrm{pk}}\right)}\right) \bullet\left(1+\mathrm{e}^{\left(\mathrm{Y}_{\mathrm{pk}}-\mathrm{O}_{\mathrm{pk}}\right)^{2}}\right)
$$

\section{* Assumption 3:}

From equations (7) and (9), $O B P$ assumed that:

$\mathrm{A}=\mathrm{A} 1 * \mathrm{~A} 2$

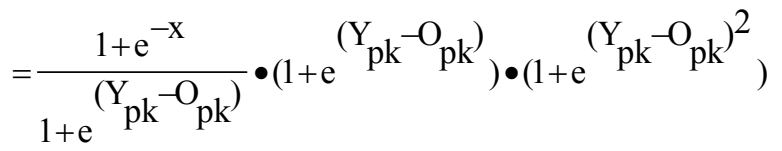

$\mathrm{A}=\left(1+\mathrm{e}^{-\mathrm{X}}\right) \cdot\left(1+\mathrm{e}^{\mathrm{Y}_{\mathrm{pk}}-\mathrm{O}_{\mathrm{pk}}}\right)^{2}$

\section{Comparative Study}

The $O B P$ will be tested using the following real example that consider neural network with 6 units for input layer, 4 units for hidden layer, and 3 units for output layer. This example will train the network using $O B P$, and then it trains it using standard $B P$, and it compares the final results from $O B P$ and $B P$.

After the MSE (Mean Square Error) reached to 0.001 the training process discontinued. The initial weights selected randomly from -0.5 to +0.5 , and the same initial weights have been used for the two algorithms. Learning rate equals to 0.01 has been taken in the training process Figure 2, shows the differences between the final weights from input layer to the hidden layer using an $O B P$, and $B P$ are very small. 


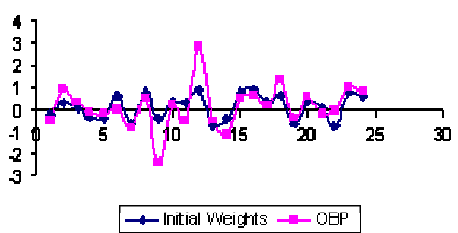

(a) Adapting process of weights from input to hidelen Jayrer using $O B P$.
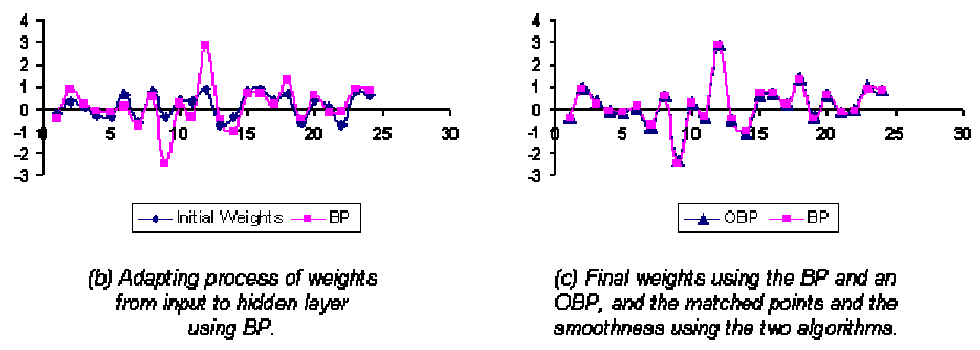

(c) Final weights using the $B P$ and an OBP, and the matched points and the smoothness using the two algorithms.

Figure 2 - Final Weight Values From Input to Hidden

Also, there are no huge differences between the two algorithms to adapt the weights from the hidden to the output layer. Figure (3) shows that:
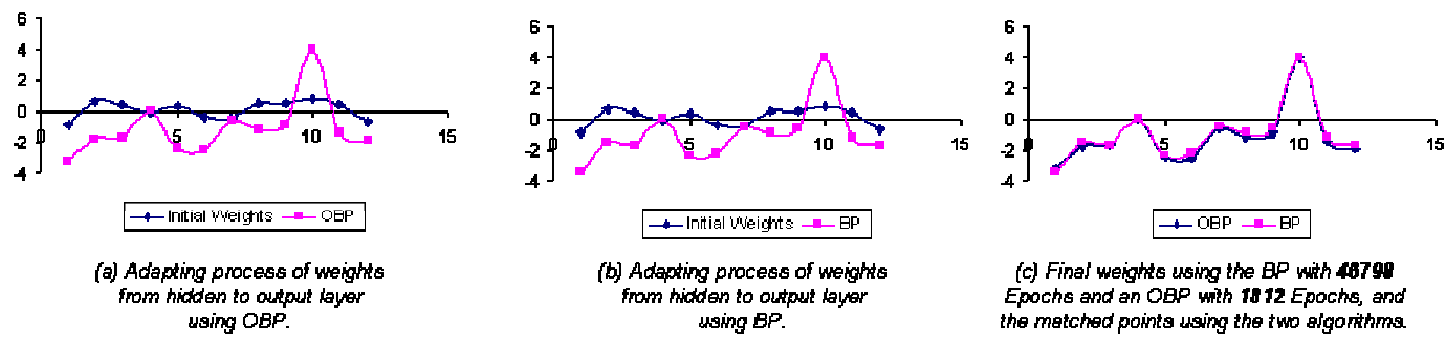

Figure 3 - Final Weight Values From Hidd en to Output

The surprising results in the previous figure are the number of epochs using the two algorithms, which proves that the $O B P$ is an optical algorithm if it is compared with the standard $B P$.

This network was tested using the two algorithms using different learning rate, Table 1 , shows the results.

From Table1, it is observed that the results of OBP are much better and faster than the BP for all training processes with different learning rate.

\section{Conclusion}

This paper introduced a new algorithm OBP, which has been proposed for the training of multilayer neural networks, and it enhanced the version of the Backpropagation BP algorithm. The study shows that OBP is beneficial in speeding up the learning process. The simulation results confirmed these observations.

Training process defined as adapting weights for each unit in neural network, so the OBP is a good algo-

\begin{tabular}{|c|c|c|}
\hline \multicolumn{3}{|c|}{$\begin{array}{c}\text { Table 1- Training processes } \\
\text { using different }(\boldsymbol{\eta})\end{array}$} \\
\hline $\begin{array}{c}\text { Learning Rate } \\
(\boldsymbol{\eta})\end{array}$ & $\begin{array}{c}\boldsymbol{O B P} \\
\text { Epochs }\end{array}$ & $\begin{array}{c}\boldsymbol{B P} \\
\text { Epoch }\end{array}$ \\
\hline 0.01 & 1812 & 46798 \\
\hline 0.05 & 363 & 9351 \\
\hline 0.1 & 182 & 4673 \\
\hline 0.15 & 122 & 3114 \\
\hline 0.2 & 92 & 2334 \\
\hline 0.25 & 47 & 1866 \\
\hline 0.3 & 61 & 1554 \\
\hline
\end{tabular}
rithm, because it can adapt all weights with optical time. The simulation results show that when a very small value is used for learning rate $(\eta)$ with OBP makes the adapted final weights very closed become to the final weights that introduced from BP. So, it can escape from local minim.

\section{Future Work}

Future work will test the proposed algorithm across a wide range of important problems and applications. 


\section{Appendix: Training a Feed Forward Neural Networks Using the Backpropagation Algorithm}

Assume there are $m$ input units, $n$ hidden units, and $p$ output units.

1. Apply the input vector, $\mathrm{X}_{\mathrm{p}}=\left(X_{p 1}, X_{p 2}, X_{p 3}, \ldots ., X_{p N}\right)^{\mathrm{t}}$ to the input units .

2. Calculate the net- input values to the hidden layer units:

$n e t_{p j}^{h}=\left(\sum_{i=1}^{N} W^{h} j i \bullet X{ }_{p i}\right)$

3. Calculate the outputs from the hidden layer:

${ }^{i} p j=f^{h}\left(n e t^{h}{ }_{p j}\right)$

4. Move to the output layer. Calculate the net-input values to each unit:

${ }_{n e t}{ }^{O} p k=\left(\sum_{j=1}^{L} W_{k j} O_{k j}\right)$

5. Calculate the outputs:

$$
O_{p k}=f^{o}\left(\text { net }^{o} p k\right)
$$

6. Calculate the error terms for the output units:

$\delta^{o}{ }_{p k}=\left(Y_{p k}-O_{p k}\right) \cdot f^{o^{\prime}} k\left(n e t^{o} p k\right)$

Where,

$f^{o^{\prime}}{ }_{k}\left(n e t_{p k}^{o}\right)=f^{o}{ }_{k}\left(n e t_{p k}^{o}\right) \bullet\left(1-f^{o}{ }_{k}\left(n e t_{p k}^{o}\right)\right)$

7. Calculate the error terms for the hidden units

$\delta_{p j}^{h}=f^{h^{\prime}}\left(n e t^{h}{ }_{p j}\right) \bullet\left(\sum_{K=1}^{M} \delta_{p k}^{o} \bullet W_{k j}^{o}\right)$

Notice that the error terms on the hidden units are calculated before the connection weights to the output-layer units have been updated.

8. Update weights on the output layer

$$
W^{o}{ }_{k j(t+1)}=W^{o} k j(t)+\left(\eta \bullet \delta^{o} p k \bullet i_{p j}\right)
$$

9. Update weights on the Hidden layer

$$
W^{h} j i(t+1)=W^{h} j i(t)+\left(\eta \bullet \delta^{h} p j \bullet X_{i}\right)
$$

\section{References}

Callan, R. (1999). The Essence of Neural Networks, 33-52. Southampton Institute.

Carling, A., (1992). Back propagation. Introducing Neural Networks, 133-154.

Caudill, M. \& Butler, C. (1993).Understanding neural networks. Computer Explorations, 1,155-218. 
Fahlman, S. E. (1988). Faster-learning variations on backpropagation: An empirical study. Proceedings of the 1988 Connectionist Models Summer School, 38-51.

Freeman, J. A. \&, Skapura, D. M. (1992). Backpropagation. Neural Networks Algorithm Applications and Programming Techniques, 89-125.

Hagan, M. T. \& Demuth, H. (1996). Neural Networks Design, 11.1-12.52.

Hakin, S. (1999). Neural networks: A comprehensive foundation ( $2^{\text {nd }}$ ed.). pp.161-184

Jacobs, R. A. (1988). Increased rates of convergence through learning rate adaptation. Neural Networks, 1 , $169-180$

Lee, Y., Oh, S. H., \& Kim, M.W. (1991). The effect of initial weights on premature saturation in back propagation learning. Proceedings of the International Joint Conference on Neural Networks, Seattle, $1765-1770$

Minai, A. A., \& Williams, R. D (1990). Acceleration of back-propagation through learning rate momentum adaptation. Proceedings of the International Joint Conference on Neural Networks, 1676-1679.

Otair, M. A. \& Salameh, W. A. (2004). An improved back-propagation neural networks using a modified non-linear function. Proceedings of the IASTED International Conference, 2004, 442-447.

Riedmiller, M \& Braun, H. (1993). A direct adaptive method for faster backpropagation learning the PROP algorithm. Proceedings of the IEEE international conference on Neural Networks (ICNN), Vol. I, San Francisco, CA, 586-591.

Rumelhart, D. E., Durbin, R. Golden, R., \& Chauvin, Y. (1992). Backpropagation: Theoretical foundations. In Y.Chauvin \& D. E Rumelhart (Eds.), Backpropagation and Connectionist Theory. Lawrence Erlbaum.

Rumelhart, D. E., Hinton, G. E., \& Williams, R. J. (1986). Learning internal representations by error propagation. In D. E. Rumelhart \& J. L. McClelland (Eds.), Parallel Distributed Processing, pp. 318362.

\section{Biographies}

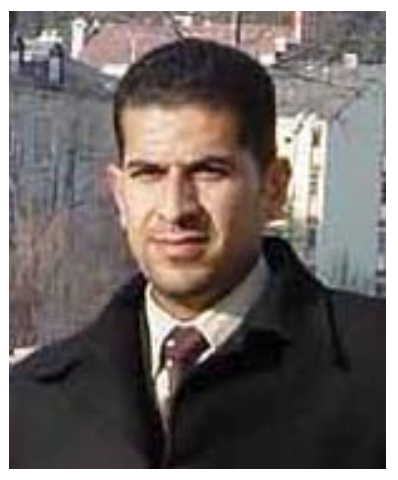

Mohammed A. Otair is an Instructor of computer information systems, at the Jordan University of Science and Technology, IrbedJordan. He received his B.Sc. in Computer Science from IU-Jordan, and his M.Sc. and Ph.D in 2000, 2004, respectively, from the Department of Computer Information Sysems-Arab Academy. His major interests are Machine Learning, Neural Network Learning Paradigms, Web-computing, E-Learning.

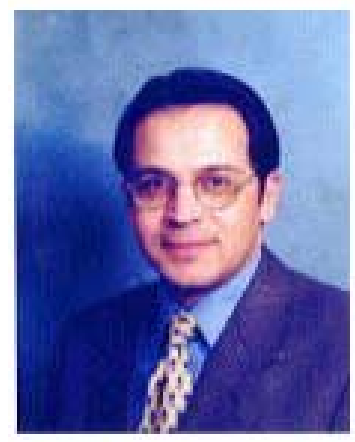

Walid A. Salameh is an Associate Professor of computer Science, at the PSUT-RSS, Amman-Jordan. Hereceived his B.Sc. in Computer Science from YU-Jordan, and his M.Sc. and Ph.D in 1987, 1991, respectively, from the Department of Computer Engineering-METU. His major interests are Machine Learning, Neural Network Learning Paradigms, and Intelligent Learning Paradigm through the Web (Intelligent Web-learning based Paradigms). 